\title{
CONTRIBUTION A LA BIOLOGIE DE PERIPATUS ACACIOI MARCUS ET MARCUS. II. VARIATIONS DU POIDS DES ANIMAUX EN FONCTION DU SEXE ET DU NOMBRE DES LOBOPODES
}

\author{
SYLVIA CAMPIGLIA * \\ et ROGER LAVALLARD **
}

* Département de Physiologie de l'Institut des Sciences Bio-médicales de l’Université de São Paulo, C.P. 4365, São Paulo, Brésil. ** Département de Physiologie de l'Institut de Biosciences de l'Université de São Paulo et Laboratoire de Physiologie des Insectes de l'Université de Paris VI. C.P 11230, São Paulo, Brésil.

CONTRIBUIÇÃO A BIOLOGIA DE PERIPATUS ACACIOI MARCUS E MARCUS. II - VARIAÇÕES DO PESO DOS ANIMAIS EM FUNÇÃO DO SEXO E DO NÚMERO DE LOBÓPODOS

\section{RESUMO}

1 - No conjunto da amostragem, a maioria dos animais tem um peso inferior a $900 \mathrm{mgr}$ e a distribuição de frequência dos pesos é bimodal, com um modo entre 100 e 199 mgr e um modo entre 400 e $499 \mathrm{mgr}$.

2 - A distribuição de frequência dos pesos considerada separadamente nos machos e nas fêmeas, é unimodal. O valor modal aproximado é, para os machos, de $169 \mathrm{mgr}$ e para as fêmeas, de $455 \mathrm{mgr}$, daí a distribuição de frequência bimodal dos pesos no conjunto da amostragem. As fêmeas são então mais pesadas do que os machos, da ordem de 2,7 vezes.

3 - As distribuições de frequência dos pesos consideradas separadamente nos machos de 25 e 26 pares de lobópodos, são unimodais. O recobrimento dos polígonos e a distribuição unimodal de frequência dos pesos para o conjunto dos machos, mostram que o número de lobópodos não tem influência sobre o peso dos indivíduos.

4 - As distribuições de frequência dos pesos consideradas separadamente nas fêmeas de 27 e 28 pares de lobópodos apresentam irregularidades provavelmente ligadas às gestações e aos partos. Entretanto, o recobrimento dos polígonos e a distribuição unimodal de frequência dos pesos para o conjunto das fêmeas estabelecem que, como nos machos, o número de lobópodos não tem influência sobre o peso dos indivíduos.

Número especial em homenagem ao Prof. Dr. Paulo Sawaya, no ano jubilar de seu magistério. 


\section{RESUME}

1 - Dans l'ensemble de l'échantillonnage, la plupart des animaux ont un poids inférieur à $900 \mathrm{mgr}$ et la distribution de fréquence des poids est bimodale, avec un mode entre 100 et $199 \mathrm{mgr}$ et un mode entre 400 et 499 mgr.

2 - La distribution de fréquence des poids considérée séparément chez les mâles et les femelles, est unimodale. La valeur modale approchée est, pour les mâles, de $169 \mathrm{mgr}$ et pour les femelles, de $455 \mathrm{mgr}$, d'où la distribution de fréquence bimodale des poids dans l'ensemble de l'échantillonnage. Les femelles sont donc beaucoup plus lourdes que les mâles, de l'ordre de 2,7 fois.

3 - Les distributions de fréquence des poids considérées séparément chez les mâles à 25 et 26 paires de lobopodes, sont unimodales. Le recouvrement de leurs polygones et la distribution unimodale de fréquence des poids pour l'ensemble des mâles, montrent que le nombre des lobopodes n'a pas d'influence sur le poids des individus.

4 - Les distributions de fréquence des poids considérées séparément chez les femelles à 27 et 28 paires de lobopodes présentent des irrégularités sans doute liées aux gestations et aux parturitions. Cependant, le recouvrement de leurs polygones et la distribution unimodale de fréquence des poids pour l'ensemble des femelles établissent que, comme chez les mâles, le nombre des lobopodes n'a pas d'influence sur le poids des individus.

Après avoir examiné, dans une population de Peripatus acacioi comprenant plusieurs centaines d'individus, le pourcentage des sexes et les variations du nombre des lobopodes (Lavallard et Campiglia, 1973), nous abordons maintenant l'étude de la taille des animaux dans cette même population ${ }^{(1)}$.

\section{MATERIEL ET METHODE}

Les données concernant l'identification du matériel utilisé ont été exposées dans le précédent travail.

La méthode d'étude de la taille des animaux doit résoudre le problème de la mesure des Onychophores. En effet, pour comparer les divers exemplaires de Peripatus acacioi, ou pour suivre les différentes phases de la croissance d'un même exemplaire, il faut pouvoir mesurer facilement chaque animal avec un système d'unités qui correspond à. des conditions semblables pour tous les animaux considérés. Cela n'a ainsi pas grande signification d'utiliser les dimensions linéaires pour mesurer des Onychophores car, comme Moseley (1874) et Oakley (1883) l'avaient déjà noté, leur corps est doué d'une telle capacité d'extension qu'il peut varier, en longueur, du simple au double. Pour 
caractériser la taille de chaque espèce en taxonomie, Bouvier (1905) insiste sur l'imprécision des dimensions linéaires des spécimens de collection, tout en justifiant leur emploi faute de meilleurs moyens, tandis que Dakin (1914) conseille de s'en défier. Claude-Joseph (1928) fournit quelques exemples de longueur et de largeur d'animaux au maximum de l'extension ou du raccourcissement. Reprenant ce problème à propos de Peripatopsis, Manton (1938) adopte, comme solution, de relever deux mesures de longueur sur l'animal vivant, l'une pendant la locomotion qui provoque une extension du corps, l'autre pendant une période d'arrêt où le corps se trouve en raccourcissement. Tout en donnant une meilleure définition des proportions des animaux, ce procédé reste d'une trop grande imprécision. En outre, peu pratique, il ne peut convenir à la manipulation d'un élevage de plusieurs centaines d'exemplaires.

Nous avons donc renoncé aux dimensions linéaires, préférant utiliser les unités de masse pour mesurer les animaux en élevage. Manton (1938) critique très justement l'emploi du poids qui peut varier de $30 \%$ pour un même animal, en fonction de l'humidité ambiante. Mais comme nous le préciserons dans un travail ultérieur traitant de la technique d'élevage, les animaux sont tous maintenus, de façon permanente, dans des conditions comparables d'humidité voisines de la saturation. Nous avons ainsi exprimé la taille des Péripates par leur poids, déterminé au milligramme près, avec suffisamment de rapidité pour éviter une erreur due à la perie d'eau des animaux pendant le pesage.

\section{RESULTATS}

\section{A. Poids des animaux dans l'ensemble de l'échantillonnage}

Il n'existe que quelques données éparses et imprécises, dans la littérature, sur la taille des Onychophores; aucun relevé portant sur une population nombreuse n'a encore été présenté. Les résultats suivants concernent 661 exemplaires de Peripatus acacioi, pour la distribution desquels nous avons divisé l'échelle des poids en classes de 100 mgr d'intervalle. La fréquence et le pourcentage de chaque classe sont donnés dans le tableau 1 et l'histogramme de fréquence se trouve sur la figure 1. 
La plupart des animaux de l'échantillonnage ont donc un poids inférieur à $900 \mathrm{mgr}$. Les exemplaires de plus d'un gramme sont rares. La courbe de distribution de fréquence des poids est bimodale. Le premier mode, compris dans la classe de 100 à $199 \mathrm{mgr}$, correspond à un polygone haut et étroit, à base contenue dans les trois premières classes. Le second mode au contraire, situé au niveau de la classe 400 à 499 mgr, appartient à un polygone aplati et large, dont la base s'échelonne sur sept classes de poids.

TABLEAU I

Fréquence des poids dans l'ensemble de l'échantillonnage. L'échelle des poids est divisée en classes de 100 mgr d'intervalle

\begin{tabular}{c|c|c}
\hline Classes de 100 mgr & Fréquence & Pourcentage \\
\hline $0-99$ & 98 & $14,83 \%$ \\
$100-199$ & 205 & $31,01 \%$ \\
$200-299$ & 99 & $14,98 \%$ \\
$300-399$ & 46 & $6,96 \%$ \\
$400-499$ & 61 & $9,23 \%$ \\
$500-599$ & 53 & $8,02 \%$ \\
$600-699$ & 32 & $4,84 \%$ \\
$700-799$ & 29 & $4,39 \%$ \\
$800-899$ & 23 & $3,48 \%$ \\
$900-999$ & 7 & $1,06 \%$ \\
$1000-1099$ & 2 & $0,30 \%$ \\
$1100-1199$ & 2 & $0,30 \%$ \\
$1200-1299$ & 2 & $0,30 \%$ \\
$1300-1399$ & 2 & $0,30 \%$ \\
& 661 & \\
\hline
\end{tabular}

B. Poids des animaux en fonction des sexes

Dans le travail fondamental de Balfour (1883), on peut déjà lire: "The sexes are distinct, and the average size of the females appears to be greater than that of the males" Cette différence sexuelle de taille est confirmée peu après par Gaffron (1885) et par Sedgwick (1888) tandis que Steel (1896) précise que les femelles ont généralement 33 à $50 \%$ de longueur en plus que les mâles et sont également plus grosses. Cette différence de taille entre les sexes est ensuite sou- 


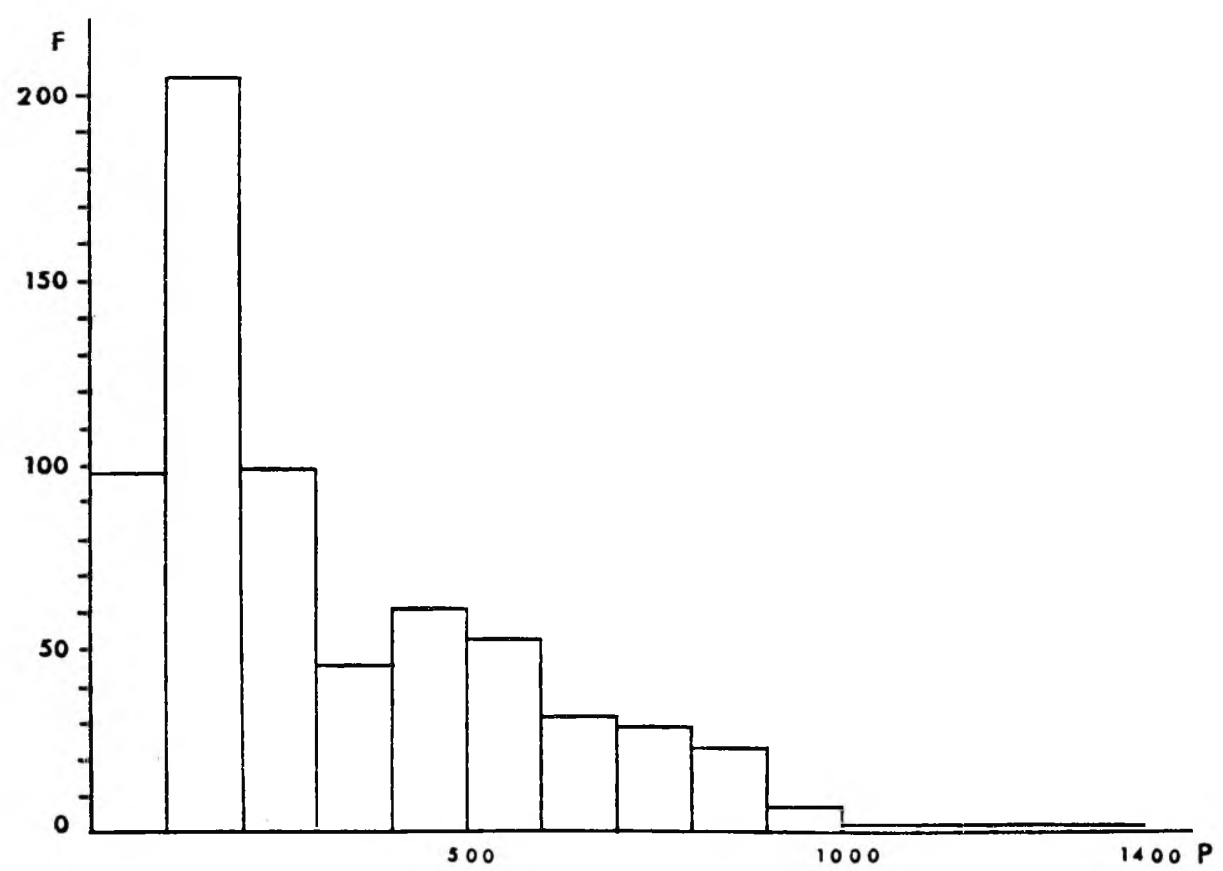

Fig. 1 - Histogramme de fréquence des poids dans l'ensemble de l'échantillonnage. F: fréquence; $\mathrm{P}$ : poids en classes de $100 \mathrm{mgr}$ d'intervalle.

vent évoquée par les auteurs, mais jusqu'à présent, aucune étude numérique n'est venue en illustrer l'observation. Nous avons donc examiné la distribution de fréquence des poids séparément, chez les mâles et les femelles.

\section{a) Chez les mâles}

Les mâles étant de dimensions bien inférieures à celles des femelles, pour l'échelle des poids, nous avons utilisé un intervalle de classe plus petit, égal à $50 \mathrm{mgr}$. Le tableau 2 rapporte la fréquence et le pourcentage de ces classes pour 301 exemplaires mâles. Dans l'histogramme de fréquence correspondant aux mâles, sur la figure 2, en raison de la diminution de moitié de l'intervalle des classes, nous avons dû multiplier par 2 l'échelle des fréquences de l'ordonnée. I] faut en effet que la somme des aires des rectangles de deux classes voisines de $50 \mathrm{mgr}$ d'intervalle, soit égale à l'aire du rectangle correspondant à ces deux classes dans un histogramme où les classes ont un intervalle de $100 \mathrm{mgr}$. 


\section{TABLEAU 2}

Fréquence des poids chez les mâles de l'échantillonnage. L'échelle des poids est divisée en classes de 50 mgr d'intervalle.

\begin{tabular}{c|c|c}
\hline Classes de $50 \mathrm{mgr}$ & Fréquence & Pourcentage \\
\hline \hline $0-49$ & 16 & $5,32 \%$ \\
$50-99$ & 44 & $14,62 \%$ \\
$100-149$ & 73 & $24,25 \%$ \\
$150-199$ & 104 & $34,55 \%$ \\
$200-249$ & 45 & $14,95 \%$ \\
$250-299$ & 17 & $5,65 \%$ \\
$300-349$ & 0 & $0,00 \%$ \\
$350-399$ & 2 & $0,66 \%$ \\
& 301 & \\
\hline
\end{tabular}

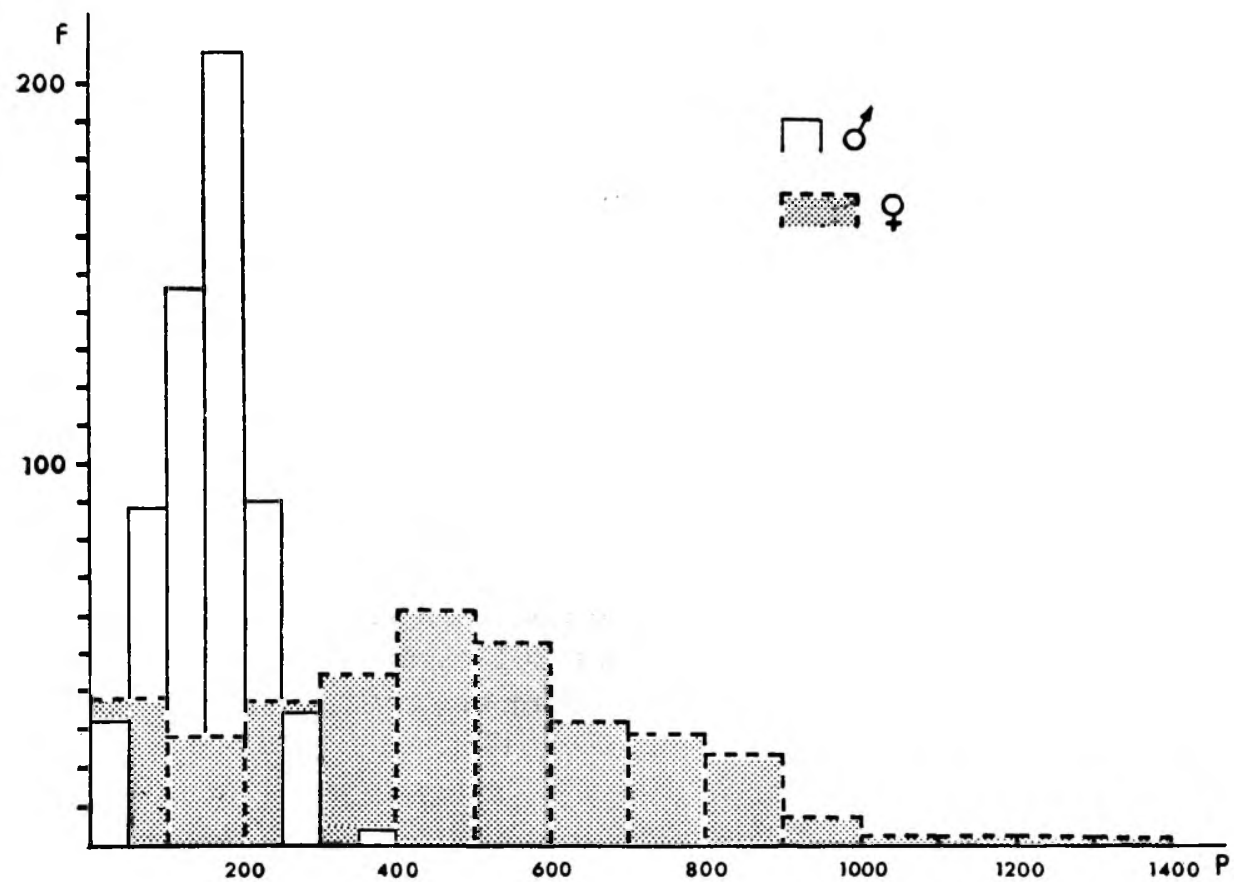

Fig. 2 - Histogramme de fréquence des poids chez les mâles et les femelles. $\mathrm{F}$ : tréquence; $\mathrm{P}$ : poids en classes d'intervalle de $50 \mathrm{mgr}$ pour les mâles et de $100 \mathrm{mgr}$ pour les femelles. 
La distribution de fréquence du poids des mâles est unimodale, suivant un polygone haut et étroit, dont le mode se situe dans la classe comprise entre 150 et 199 mgr. Etant donné que cet échantillonnage de population comprend les jeunes en pleine croissance, il nous a semblé préférable d'exprimer la taille des mâles ou des femelles par la valeur modale des poids plutôt que par leur moyenne. Selon Stanley (1963), une bonne approximation de la valeur modale s'obtient par la formule:

$$
\mathrm{M}_{0}=\mathrm{L}_{\mathrm{mo}}+\frac{\mathrm{F}_{\mathrm{a}}}{\overline{\mathrm{F}_{\mathrm{a}}+\mathrm{F}_{\mathrm{b}}}}
$$

où $\mathrm{M}_{\mathrm{o}}=$ valeur modale; $\mathrm{L}_{\mathrm{mo}}=$ limite inférieur de la classe modale; $\mathrm{F}_{\mathrm{a}}=$ fréquence de la classe immédiatement au-dessus de la classe modale; $F_{b}=$ fréquence de la classe immédiatement au-dessous de la classe modale; $\mathrm{C}=$ intervalle de classe.

Appliquée au cas des mâles de Peripatus acacioi, cette formule devient:

$$
M_{o}=150+\frac{45 \times 50}{45+73}=169 \mathrm{mgr}
$$

\section{b) Chez les femelles}

Nous avons examiné la distribution de fréquence des poids groupés en classes de 100 mgr d'intervalle chez 360 femelles. Le tableau 3 présente les données numériques de cette distribution et de son pourcentage. L'histogramme correspondant se trouve sur la figure 2.

La distribution de fréquence du poids des femelles est unimodale suivant un polygone aplati et large, dont le mode coïncide avec la classe comprise entre 400 et 499 mgr. L'application de la formule du calcul de la valeur modale donne cette fois:

$$
\mathrm{M}_{\mathrm{o}}=400+\frac{53 \times 100}{53+44}=455 \mathrm{mgr}
$$

Les femelles sont donc 2,7 plus lourdes que les mâles. Cette différence sexuelle de poids explique la distribution bimodale observée sur l'histogramme de la figure 1 où les mâles, de petite taille, sont groupés au niveau des trois premières classes, tandis que les femelles, plus grandes, sont aussi dispersées sur un plus grand nombre de classes, du fait des oscillations de poids en relation avec la gestation et la parturition. 


\section{TABLEAU 3}

Fréquence des poids chez les femelles de l'échantillonnage. L'échelle des poids est divisée en classes de $100 \mathrm{mgr}$ d'intervalle.

\begin{tabular}{c|c|c}
\hline Classes de $100 \mathrm{mgr}$ & Fréquence & Pourcentage \\
\hline $0-99$ & 38 & $10,55 \%$ \\
$100-199$ & 28 & $\mathbf{7 , 7 8 \%}$ \\
$200-299$ & 37 & $10,28 \%$ \\
$300-399$ & 44 & $12,22 \%$ \\
$400-499$ & 61 & $16,94 \%$ \\
$500-599$ & 53 & $14,72 \%$ \\
$600-699$ & 32 & $8,89 \%$ \\
$700-799$ & 29 & $8,05 \%$ \\
$800-899$ & 23 & $6,39 \%$ \\
$900-999$ & 7 & $1,94 \%$ \\
$1000-1099$ & 2 & $0,56 \%$ \\
$1100-1199$ & 2 & $0,56 \%$ \\
$1200-1299$ & 2 & $0,56 \%$ \\
$1300-1399$ & 2 & $0,56 \%$ \\
& 360 & \\
\hline
\end{tabular}

C. Poids des animaux et nombre de lobopodes dans chaque sexe

Les deux sexes montrant donc des différences dans le nombre de paires de lobopodes et dans la taille, il reste à voir, pour chaque sexe, si le nombre des lobopodes a une influence sur la taille des animaux. Nous avons ainsi établi la distribution de fréquence des poids pour chacun des nombres possibles de paires de lobopodes, séparément chez les mâles et les femelles.

\section{a) Chez les mâles}

Il fut indiqué précédemment que chez les mâles, les nombres possibles de paires de lobopodes sont 24, 25, 26 et 27 . Pour chacun, le tableau 4 rapporte la distribution de fréquence des poids dont l'échelle est divisée en classes de $50 \mathrm{mgr}$ d'intervalle. Nous avons encore calculé, pour chaque classe, le pourcentage partiel, c'est-à-dire vis-à-vis des mâles possédant seulement le nombre considéré de paires de lobopodes, et le pourcentage total, c'est-à-dire de l'ensemble des mâles examinés, soit 301 exemplaires. Laissant de côté les mâles à 24 et 27 paires 
de lobopodes dont la quantité (respectivement 3 et 14 exemplaires) est négligeable, nous avons tracé (fig. 3) l'histogramme de fréquence des poids seulement pour les mâles à 25 et 26 paires.

On retrouve une distribution unimodale pour chaque catégorie de mâles. Il y a une différence d'une classe dans la position du mode entre les mâles à 25 paires et ceux à 26 paires de lobopodes, mais le recouvrement des polygones de fréquence suggère qu'il n'y a pas de différence sensible de poids. Cette observation corrobore les résultats du tableau 2 et de la figure 2 qui démontrent, avec des classes de 50 mgr d'intervalle, que chez l'ensemble des mâles, la distribution de fréquence est unimodale. On peut donc conclure que chez les mâles, les différences dans le nombre de paires de lobopodes n'ont pas de répercussions sur la taille des individus.

\section{b) Chez les femelles}

Les nombres possibles de paires de lobopodes chez les femelles sont 26, 27, 28 et 29. Pour chacun, le tableau 5 présente la distribution

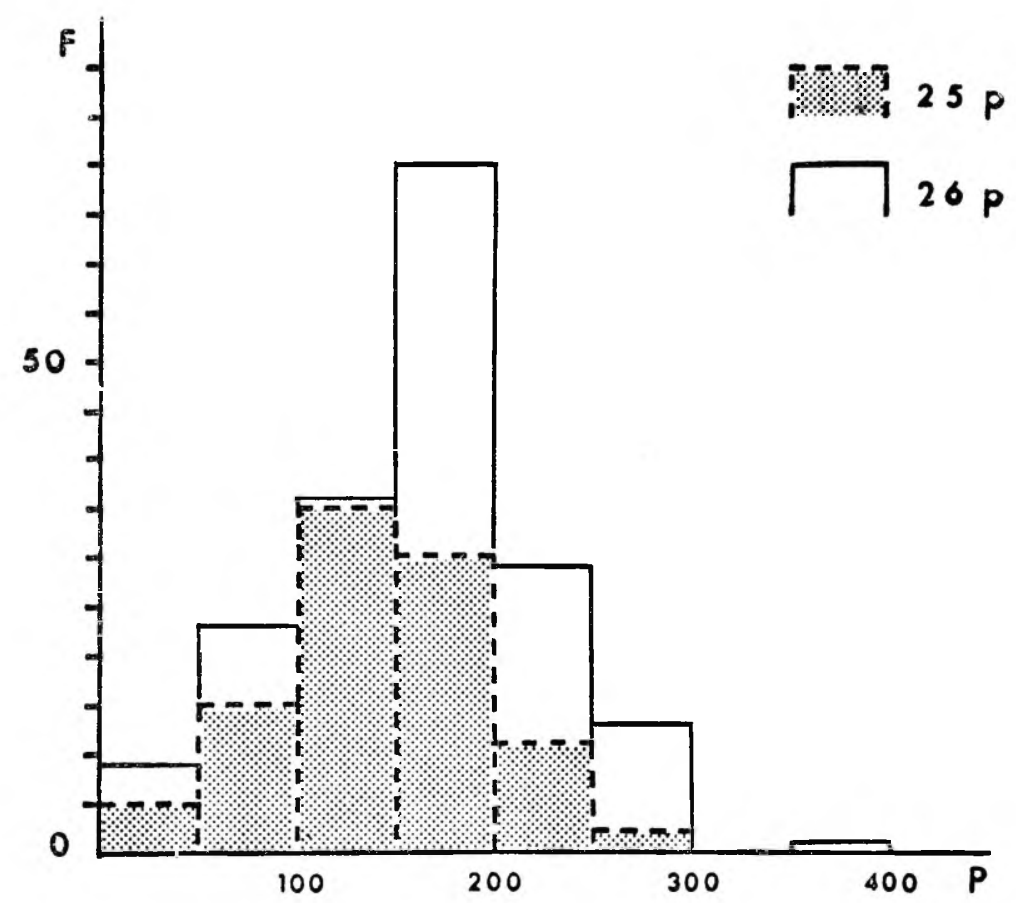

Fig. 3 - Histogramme de fréquence des poids chez les mâles à 25 et 26 paires de lobopodes. $F$ : fréquence; $P$ : poids en classes de $50 \mathrm{mgr}$ d'intervalle. 


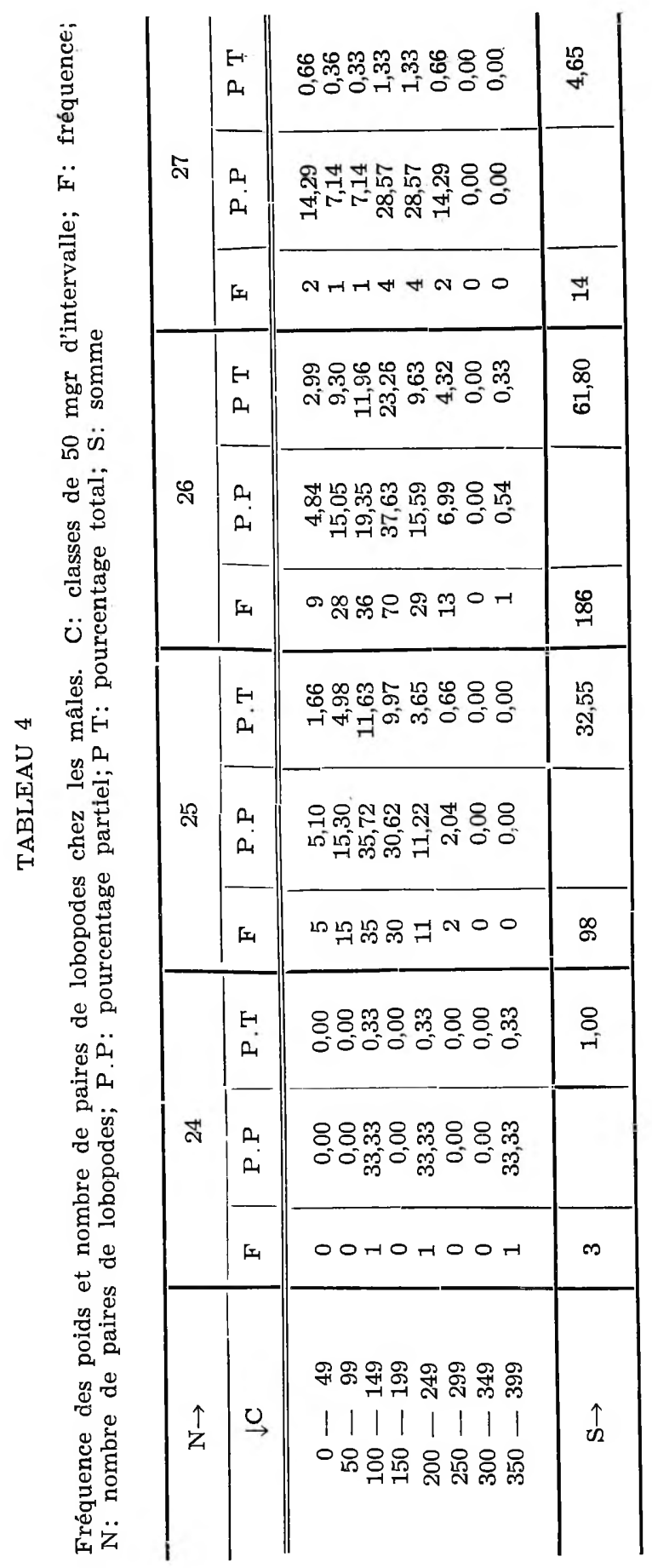




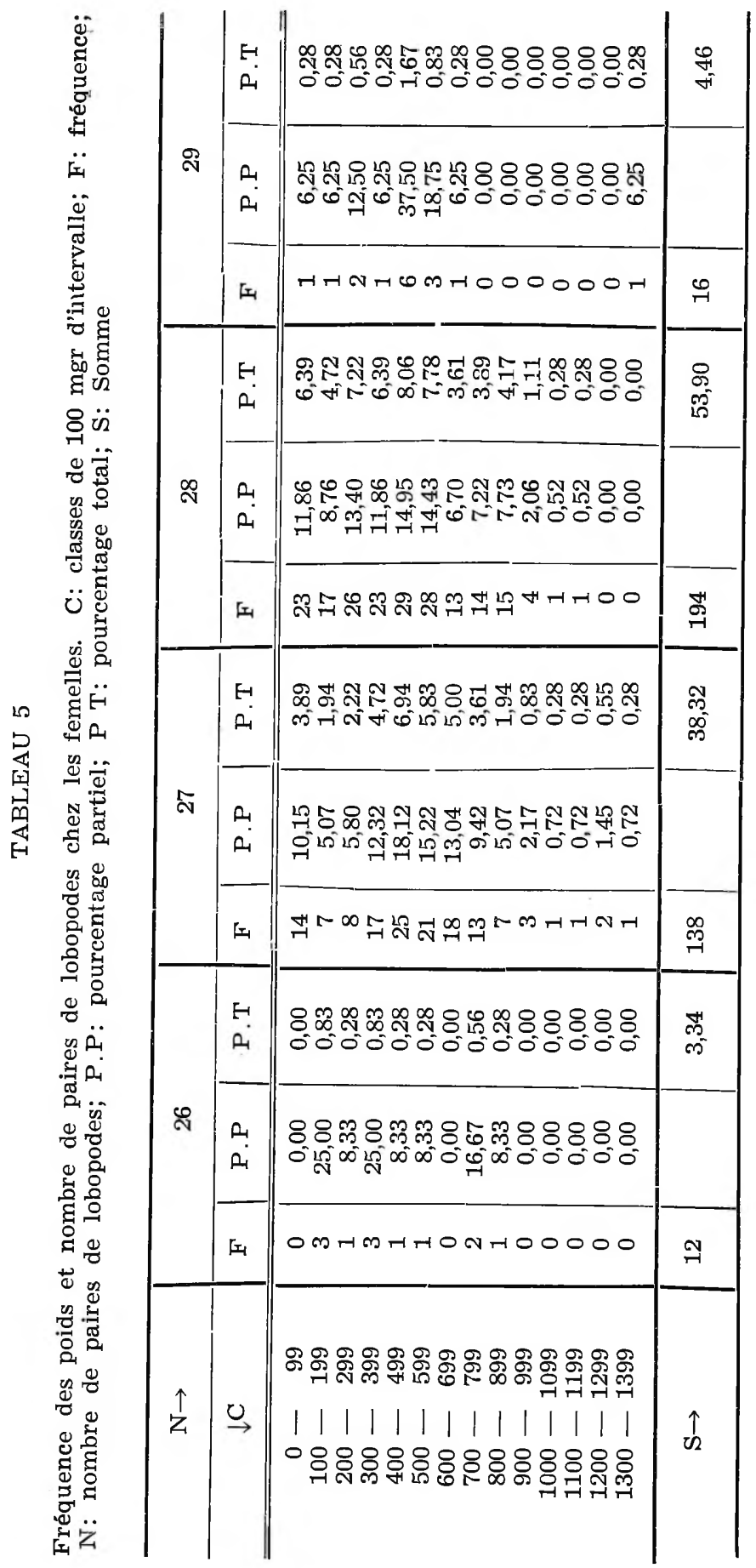


de fréquence des poids dont l'échelle est divisée en classes de $100 \mathrm{mgr}$ d'intervalle. Comme dans le cas des mâles, ce tableau donne encore, pour chaque classe, le pourcentage partiel, relatif aux femelles possédant seulement le nombre considéré de lobopodes, et le pourcentage total, concernant l'ensemble des femelles examinées, soit 360 exemplaires.

Les femelles à 26 et 29 paires d'appendices locomoteurs étant en quantité insignifiante (respectivement 12 et 16 exemplaires), nous n'avons tracé d'histogramme de fréquence (fig. 4) que pour celles à 27 et 28 paires. Chez ces dernières, dans les deux cas, la fréquence la plus grande de poids se localise au niveau de la même classe, entre 400 et 499 mgr. Cependant, d'une part, les polygones sont aplatis, d'autre part, la distribution de fréquence n'est pas unimodale et il y a même plusieurs oscillations dans le cas des femelles à 28 paires de lobopodes. Ces particularités suggèrent l'influence de facteurs autres que le nombre de lobopodes, probablement en relation avec les phénomènes de gestation et parturition.

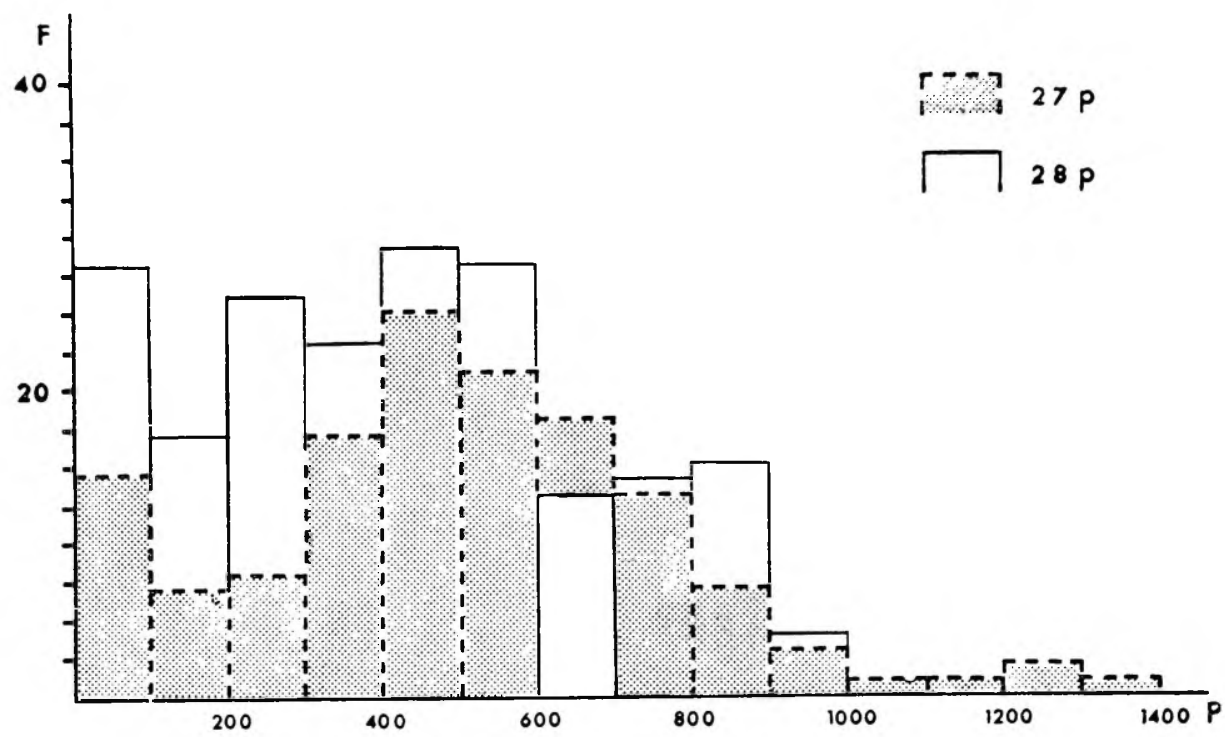

Fig. 4 - Histogramme de fréquence des poids chez les femelles à 27 et 28 paires de lobopodes. F: fréquence; P: poids en classes de $100 \mathrm{mgr}$ d'intervalle. 
Quoi qu'il en soit, dans l'histogramme de fréquence de la figure 4, les polygones des femelles à 27 et à 28 paires se recouvrent complètement. En tenant compte des résultats du tableau 3 et de la figure 2 qui montrent que la distribution de fréquence du poids des femelles est unimodale, il faut conclure qu'également chez les femelles, les différences dans le nombre de paires de lobopodes n'ont pas d'influence sur la taille des individus.

\section{DISCUSSION}

Chez Peripatus acacioi, comme d'ailleurs chez tous les Peripatidae, les différences de taille et de nombre d'appendices locomoteurs appartiennent en grande partie au dimorphisme sexuel. Pour reconnaître les mâles et les femelles sur le vivant, il n'y a, comme caractère morphologique précis, que la présence ou l'absence des papilles crurales sur les deux paires de lobopodes prégénitales. Pratiquement, c'est un critère laborieux, puisqu'il nécessite une anesthésie des animaux et un examen soigneux au fort grandissement de la loupe binoculaire. Dès lors, la connaissance des histogrammes de fréquence du nombre des paires de lobopodes et du poids dans les deux sexes, fournit un moyen rapide de séparer les mâles des femelles, pour la majorité des individus d'une récolte destinée à un élevage expérimental. En effet, tous les individus à 24 et 25 paires de lobopodes sont des mâles; tous ceux à 28 et 29 paires sont des femelles; les exemplaires à 26 et 27 paires pesant plus de $300 \mathrm{mgr}$ sont des femelles. Il ne faut donc avoir recours au critère des papilles crurales que pour les animaux de petite taille ayant 26 et 27 paires de lobopodes, ce qui réduit la tâche considérablement. Encore peut-on même, pour un élevage, considérer comme mâles les petits individus à 26 paires de lobopodes puisque ,d'une part, cela est vrai dans $94 \%$ des cas et d'autre part, la croissance permettra ultérieurement de reconnaître les éventuelles femelles. Par ailleurs, dans chaque sexe, on peut distinguer d'après la distribution de fréquence des poids, un certain nombre de catégories d'animaux en choisissant par exemple, les limites adoptées pour les intervalles des classes de poids. On verra l'importance de cette distinction ultérieurement, quand il faudra examiner la fréquence des mues en fonction de la taille des animaux. Donc, en dehors des considérations théoriques mettant en évidence quelques particularités du groupe des Onychophores, l'étude des trois paramètres, sexe, nombre de lobopodes, taille, a surtout une finalité pratique pour le reste du travail. 
Il est fort probable que les irrégularités de fréquence des poids chez les femelles à 27 et 28 paires de lobopodes, proviennent des oscillations inviduelles de poids provoquées par la gestation et la parturition des portées. Chez les femelles, pour confirmer qu'il n'y a pas une influence du nombre des lobopodes sur le poids, il faudrait avoir recours à des vierges. Or ces dernières sont fort rares dans la nature, car la fécondation est précoce. La meilleure solution serait de faire un élevage de longue durée, avec un assez grand nombre de femelles isolées dès la naissance. Cela n'a pas été fait ici parce qu'il s'agissait d'un aspect trop en dehors du cadre de ce travail, mais il n'y aurait certainement pas de difficultés majeures à le réaliser.

(1) Ce travail a été réalisé avec l'aide de la "Fundação de Amparo à Pesquisa do Estado de São Paulo"

BALFOUR, F. M. (1883) - The Anatomy and Development of Peripatus capensis. Quart. J. micr. Sci., 23:213-259.

BOUVIER, E.-L. (1905) - Monographie des Onychophores (Pr $\in$ mière partie). Ann. Sc. nat., (Zool.), 9 sér., 2:1-383.

CLAUDE-JOSEPH, H. (1928) - Observations sur un Péripate du Chili, (Opisthopatus blainvillei Gay-Gervais). Ann. Sc. nat., (Zool.), 10 sér., 11:285-298.

DAKIN, W. J. (1914) - Fauna of Western Australia. I - The Onychophora of Western Australia. Proc. zool. Soc., London: 289-292.

GAFFRON, E. (1885) - Beitrage zur Anatomie und Histologie von Peripatus. Zool. Beitr., Breslau, 1:33-60.

LAVALlARD, R. \& CAMPIGLIA, S. (1973) - Contribution à la biologie de Peripatus acacioi Marcus et Marcus. I - Pourcentage des sexes et variations du nombre des lobopodes dans un échantillonnage de plusieurs centaines d'individus. Bol. Zool. e Biol. Mar., n.s., 30:sous presse.

MANTON, S. M. (1938) - Studies on the Onychophora. VI - The life-history of Peripatopsis. Ann. Mag. nat. Hist., ser. 11, 1:515-529.

MOSELEY, H. N. (1874) - On the structure and development of Peripatus capensis. Proc. Roy. Soc., 22:344-350.

OAKLEY, H. W. (1883) - On Peripatus capensis. Trans. S. Afr. phil. Soc., 3:35-37.

SEDGWICK, A. (1888) - A Monograph on the Species and Distribution of the Genus Peripatus (Guilding). Quart. J. micr. Sci., 28:431-493.

STANLEY, J. (1863) - The essence of biometry. Mc Gill University Press, Montreal.

STEEL. T. (1896) - Observations on Peripatus. Proc. linn. Soc. N. S. W., 21: S4-103. 